\title{
Opinião de adolescentes sobre as características essenciais ao cirurgião-dentista: estudo em municípios sul-brasileiros
}

\author{
Adolescent's opinion about the essential characteristics \\ to the dentist: study in southern brazilian municipalities
}

\author{
ELISABETE RABALDO BOTTAN ${ }^{1}$ \\ CARINI GABRIELA PIVATTO ${ }^{2}$ \\ EWELIN CRISTINE DA SILVA ${ }^{3}$ \\ LETICIA WESTPHAL BESEN ${ }^{4}$ \\ LUCIANE CAMPOS 5 \\ CONSTANZA MARÍN ${ }^{6}$
}

\begin{abstract}
RESUMO
Objetivo: Analisar a visão de adolescentes sobre as características essenciais ao exercício da profissão de cirurgião-dentista. Método: Estudo exploratório com abordagem quali-quantitativa. A população-alvo constituiu-se de alunos do $9^{\circ}$. ano, matriculados em escolas do perímetro urbano de três municípios de Santa Catarina (Brasil). A coleta de dados ocorreu com base nos princípios da Técnica de Associação Livre de Palavras. As diversas evocações mencionadas pelos pesquisados foram listadas e classificadas em duas categorias, de acordo com suas afinidades conceptuais. Para verificar a associação entre categorias de análise e os fatores sexo e cidade de procedência, foi adotado o teste do qui-quadrado $(p \leq 0,01)$.

Resultados: Participaram do estudo 436 adolescentes (51\% do sexo feminino), com idade média de 14,68 anos. Foram consideradas 1.182 evocações, sendo 70,8\% sobre Competências Técnico-Científicas e $29,2 \%$ sobre Relações Interpessoais. A categoria Competências Técnico-Científicas, além de ser a mais frequente foi a mais citada em primeira ordem de evocação. A análise evidenciou associações significativas, havendo uma maior diferença entre as categorias em dois dos três municípios. A relação entre as categorias, segundo sexo e município, indica comportamentos diferenciados. No município $A$, a diferença foi maior entre adolescentes do sexo feminino; no município B entre os do sexo masculino; e no município $\mathrm{C}$ houve similaridade.

Conclusão: Para esse grupo de adolescentes, as características essenciais ao cirurgião-dentista estão fortemente vinculadas à excelência do fazer técnico-científico, sem desconsiderar, no entanto, as características que envolvem as relações interpessoais.
\end{abstract}

Palavras-chave: Adolescente. Recursos Humanos em Odontologia. Relações Dentista-Paciente. Satisfação do Paciente.

\section{ABSTRACT}

Objective: To analyze the adolescent's view about the essential characteristics to the exercise of dentist profession.

Method: Exploratory study with qualitative and quantitative approach. The target population consisted of $9^{\text {th }}$ graders enrolled in schools within the urban area in three cities of Santa Catarina (Brazil). Data collection was carried out based on the principles of the Free Word Association Technique. The numerous evocations

\footnotetext{
Professora do Grupo de Pesquisa Atenção à Saúde Individual e Coletiva em Odontologia do Curso de Odontologia da Universidade do Vale do Itajaí.

2 Acadêmica e Bolsista de Iniciação Científica do Curso de Odontologia da Universidade do Vale do Itajaí.

Acadêmica e Bolsista de Iniciação Científica do Curso de Odontologia da Universidade do Vale do Itajaí.

Cirurgiã-dentista egressa do Curso de Odontologia da Universidade do Vale do Itajaí.

5 Professora do Grupo de Pesquisa Atenção à Saúde Individual e Coletiva em Odontologia do Curso de Odontologia da Universidade do Vale do Itajaí.

6 Professora do Grupo de Pesquisa Atenção à Saúde Individual e Coletiva em Odontologia do Curso de Odontologia da Universidade do Vale do Itajaí.
} 
mentioned by the subjects were listed and classified in two categories, according to conceptual similarity. To verify the association between categories of analysis and gender and hometown, the Chi-square test was used ( $\mathrm{p} \leq 0.01)$.

Results: The study included 436 adolescents (51\% were female) with an average age of 14.68 years. A total of 1182 evocations were considered, among which $70.8 \%$ about Technical-Scientific Competencies and $29.2 \%$ about the Interpersonal Relationships. The category Technical-Scientific Competence, besides being the most frequent was most cited in the first order of evocation. The analysis showed significant association with a greater difference between categories in two of the three municipalities. The relationship between the categories, according to sex and municipality, indicates different behaviors. In city A, the difference was higher among female adolescents; in the municipality B among the males; and in county $\mathrm{C}$ there was similarity.

Conclusion: For this group of adolescents, the characteristics essential to the dental surgeon are strongly linked to the excellence of the technical-scientific work, without, however, disregarding the characteristics that involve interpersonal relations.

Keywords: Adolescent. Dental Staff. Dentist-Patient Relation. Patient Satisfaction.

\section{INTRODUÇÃO}

A adolescência é um momento em que o jovem pode adquirir conhecimentos que reforçam atitudes e comportamentos positivos, os quais persistirão no futuro, representando um momento fundamental para a promoção da saúde. Portanto, cabe aos profissionais da área da saúde, entre os quais o cirurgiãodentista, dedicar especial atenção a essa etapa da vida, uma vez que todos os cuidados dispensados aos adolescentes influenciarão no perfil de saúde bucal desses sujeitos quando na fase adulta. ${ }^{1-4}$

Os adolescentes, de acordo com as diretrizes da política de saúde brasileira, constituem um grupo prioritário para o atendimento programado. ${ }^{5}$ Contudo, cada sujeito vive esse período de modo diferenciado em decorrência de fatores sociais, culturais, temporais, entre outros, sendo necessário, por parte dos profissionais da área da saúde, um olhar específico para as particularidades. , $^{1,6-10}$ É fundamental que a prestação de serviços a esse grupo populacional considere todos esses aspectos, havendo a necessidade de mudanças para que se promova efetivamente a melhoria da saúde.

Nesse sentido, é relevante investigar opiniões, valores e crenças dos pacientes com o propósito de que, a partir da compreensão desses aspectos, o cirurgião-dentista possa contribuir com um processo de reflexão consciente sobre as reais condições de saúde de seu paciente e com a sua transformação em agente ativo das mudanças necessárias. Conhecer a imagem que usuários de serviços odontológicos têm a respeito do perfil do profissional é extremamente significante, em especial quando esse paciente for um adolescente. A caracterização elaborada pelos pacientes evidencia importantes aspectos os quais devem ser considerados durante o processo de formação profissional do cirurgiãodentista, que se inicia com a graduação e se estende ao longo de sua carreira.

Assim, este estudo teve como objetivo analisar a concepção de adolescentes quanto às características essenciais para o exercício da profissão de cirurgião-dentista, em três municípios do Estado de Santa Catarina, no sul do Brasil.

\section{Métodos}

O estudo exploratório com abordagem quali-quantitativa teve como populaçãoalvo os escolares matriculados, em 2016, no último ano do ensino fundamental ( $9^{\circ}$. ano), de onze escolas públicas localizadas em três municípios de Santa Catarina. 
A escolha dos municípios ocorreu por conveniência. O município $A$, pertencente à região da Grande Florianópolis - Santa Catarina -, caracteriza-se por uma colonização alemã e apresenta uma densidade demográfica de 32 hab. $/ \mathrm{km}^{2}$. Sua economia está suportada na agricultura familiar. O Índice de Desenvolvimento Humano - IDH - é de 0,749, considerado como alto. O município B situase na mesorregião do Vale do Itajaí, no litoral norte de Santa Catarina a, aproximadamente, $110 \mathrm{~km}$ da capital do Estado. Sua densidade demográfica é 171 hab./km². A colonização é açoriana e a base econômica consta do turismo e do comércio; o IDH é alto $(0,756)$. O município $C$, também localizado na mesorregião do Vale do Itajaí, está distante cerca de $90 \mathrm{~km}$ da capital do Estado. Possui uma densidade demográfica de 649,75 hab./ $\mathrm{km}^{2}$. Sua colonização é açoriana e a base econômica está na indústria, na pesca e na atividade portuária. O seu IDH é de 0,736 , que é considerado alto.

As escolas foram selecionadas de acordo com os seguintes critérios: localização no perímetro urbano; possuir ensino fundamental; e ser da rede pública. No município $A$, foi incluída uma escola e nos municípios $B$ e $C$ cinco, em cada um. Para composição da amostra, nos municípios $B$ e $C$ efetuou-se o sorteio de uma turma em cada escola. Para o município $A$, que contava com apenas uma escola, foram incluídas todas as turmas de nono ano.

Os critérios de inclusão dos sujeitos na amostra foram: ter idade entre 14 e 15 anos; aceitar por livre e espontânea vontade participar da pesquisa; apresentar Termo de Consentimento Livre e Esclarecido - TCLE - assinado pelo responsável e pelo próprio adolescente. Para este estudo, tomou-se a classificação de adolescente da Organização Mundial da Saúde que, também, é adotada pelo Ministério da Saúde do Brasil. ${ }^{11} \mathrm{~A}$ adolescência se estende dos 10 aos 20 anos de idade. Esse período não é homogêneo, dividindo-se em três estágios que apresentam características próprias. O início da adolescência vai dos 10 aos 13 anos de idade e o estágio final dos 16 aos 20 anos. O período da adolescência média abrange a faixa etária entre 14 e 15 anos e se caracteriza pelo desenvolvimento do senso da identidade, do relacionamento e do pensamento reflexivo. ${ }^{1} \mathrm{Em}$ decorrência dessas características, optamos por conduzir o estudo com sujeitos que se encontravam na fase média da adolescência.

Os cuidados éticos envolveram procedimentos de informação, por parte dos pesquisadores, sobre a pesquisa e obtenção da anuência dos pais e dos próprios escolares, por meio da assinatura do Termo de Consentimento Livre e Esclarecido (TCLE). Em sala de aula, os alunos receberam informações acerca da pesquisa e lhes foi entregue o TCLE para coletarem a assinatura de seus pais ou responsáveis e para que, também, assinassem o referido documento. Na data agendada de comum acordo com a direção das escolas, uma única pesquisadora, em cada cidade, aplicou o instrumento de coleta de dados. A aplicação do instrumento foi efetuada no mês de março de 2016.

As características essenciais ao exercício profissional de um cirurgião-dentista foram obtidas a partir do Teste de Associação Livre de Palavras, o qual permite colher elementos por meio de expressões verbais espontâneas menos controladas e mais autênticas, a partir de um estímulo indutor. Essa técnica evidencia universos comuns de palavras expressas por diferentes sujeitos ou grupos. ${ }^{12}$ Neste estudo, o estímulo indutor foi o seguinte questionamento: Quais características você considera mais importantes para um cirurgião-dentista exercer sua profissão?

Anteriormente à aplicação do instrumento de coleta de dados, as 
pesquisadoras orientaram os participantes, salientando que eles deveriam utilizar, preferencialmente, expressões ou palavras isoladas que lhes viessem à mente sem a preocupação com formas rebuscadas de escrita. Foi estipulado um tempo máximo de até cinco minutos para que os adolescentes registrassem suas ideias.

A organização dos dados ocorreu mediante os seguintes procedimentos: Leitura preliminar que consistiu na tomada de contato com os 436 documentos produzidos pelos adolescentes. Essa etapa teve por objetivo identificar as evocações registradas. Para tanto, foram marcadas todas as expressões compatíveis com o estudo. As ideias confusas ou não pertinentes foram descartadas; desse modo, obteve-se um total de 1.182 evocações. Posteriormente, realizou-se nova leitura para o agrupamento das evocações, por similaridade de significado. Foram preestabelecidas duas categorias (Quadro 1) com base no trabalho de Bottan et al. ${ }^{13}$

Quadro 1: Descrição das categorias.

\begin{tabular}{|c|l|}
\hline CATEGORIAS & \multicolumn{1}{|c|}{ EVOCAÇÕES } \\
\hline & $\begin{array}{l}\text { Atualizado. Participar de cursos. Fazer tratamentos de modo correto. Realizar } \\
\text { tratamentos bem-sucedidos. Fazer tratamentos modernos. Adotar medidas de } \\
\text { biossegurança, de esterilização e de higiene. Manter o ambiente de trabalho } \\
\text { organizado e limpo. Cumprir com o horário marcado. Ter equipamentos modernos... }\end{array}$ \\
\hline $\begin{array}{c}\text { Técnico-Científicas } \\
\text { Relacionamento } \\
\text { Interpessoal }\end{array}$ & $\begin{array}{l}\text { Simpático. Bom relacionamento com o paciente. Humano. Atencioso. Conhecer os } \\
\text { temores do paciente. Saber conversar com o paciente. Não discriminar o paciente. } \\
\text { Respeitar o paciente. Ser honesto. Ser amigo. }\end{array}$ \\
\hline
\end{tabular}

Após a identificação e a classificação das características, efetuou-se a quantificação das evocações de cada categoria, com o objetivo de se obter a frequência de ocorrência, segundo a cidade de procedência e o sexo dos pesquisados, permitindo-nos, assim, determinar a intensidade que os significados foram expressos. Todo esse procedimento foi realizado de modo manual e consensual entre o grupo de pesquisadores.

Os dados foram registrados em planilha do Microsoft Excel 2010. Utilizou-se o teste não paramétrico do qui-quadrado $\left(X^{2}\right)$ para identificar associação entre as categorias e as variáveis (sexo e cidade de procedência), tendo sido consideradas diferenças significativas aquelas com um "p" crítico igual ou menor que 0,01.

Esta pesquisa integrou um programa de estudos que tem por foco a saúde bucal de adolescentes e foi submetida e aprovada pelo Comitê de Ética em Pesquisa pelo Parecer $n^{\circ}$. 1.224.167 (CAAE 43010815.5.0000.0120) e todos os seus procedimentos foram conduzidos em conformidade com os preceitos éticos.

\section{Resultados}

Participaram do estudo 436 adolescentes. As características demográficas da amostra estão descritas no Quadro 2 e aquelas referentes à utilização de serviços odontológicos por esses adolescentes estão no Quadro 3. 
Quadro 2: Caracterização da amostra.

\begin{tabular}{|l|c|c|cc|}
\hline \multicolumn{1}{|c|}{ Aspectos demográficos } & Município A & Município B & Município C & Total \\
\hline Número de matriculados & 180 & 152 & 195 & 527 \\
Número de participantes & $140(78 \%)$ & $148(97 \%)$ & $148(76 \%)$ & 436 \\
Sexo Feminino & $78(56 \%)$ & $73(49 \%)$ & $73(49 \%)$ & 224 \\
Sexo Masculino & $62(44 \%)$ & $75(51 \%)$ & $75(51 \%)$ & 212 \\
Idade média & 15,10 & 14,68 & 14,27 & 14,68 \\
\hline
\end{tabular}

Quadro 3: Caracterização dos serviços odontológicos utilizados pelos adolescentes

\begin{tabular}{|l|c|c|c|}
\hline \multicolumn{1}{|c|}{ Itens } & Município A & Município B & Município C \\
\hline Tipo de Clínica: & & & \\
Particulares e Convênios & $70 \%$ & $52 \%$ & $34 \%$ \\
Unidades Básicas de Saúde & $30 \%$ & $48 \%$ & \\
\hline Última consulta: & & & $74,5 \%$ \\
Menos de um ano & $96 \%$ & $60 \%$ & $12,0 \%$ \\
Mais de um ano & $4 \%$ & $12 \%$ & $13,5 \%$ \\
Não lembra & $0 \%$ & $28 \%$ & \\
\hline Motivo da consulta: & & & $37,5 \%$ \\
Tratamento ortodôntico & $46,5 \%$ & $39,5 \%$ & $26,0 \%$ \\
Rotina/Prevenção & $32,5 \%$ & $26,0 \%$ & $21,5 \%$ \\
Dor/Cárie & $12,0 \%$ & $20,0 \%$ & $5,0 \%$ \\
Clareamento dental & $5,0 \%$ & $2,0 \%$ & $10,0 \%$ \\
Não lembra/Não informou & $4,0 \%$ & $12,5 \%$ & \\
\hline
\end{tabular}

A análise das características essenciais ao cirurgião-dentista foi efetuada com base em 420 evocações procedentes dos alunos do município A, 368 do município B e 394 do município $C$, que totalizaram 1.182 evocações.

A maioria das evocações $(70,8 \%)$ se referia à categoria Competências TécnicoCientíficas; a categoria Relações Interpessoais obteve $29,2 \%$ das citações. Adolescentes de ambos os sexos deram maior destaque às características relacionadas às Competências Técnico-Científicas, porém a frequência foi um pouco maior entre adolescentes do sexo feminino $(71,71 \%)$ do que entre os do sexo masculino $(68,44 \%)$. No entanto, pelo teste do qui-quadrado não há diferença estatisticamente significativa, ao se relacionar sexo e categorias de análise $(p=0,097)$.

Quanto à frequência das duas categorias segundo o município de procedência, identificou-se que a categoria Competências Técnico-Científicas foi mais frequente para os três grupos, com diferença estatisticamente significativa $(p=0,000)$. Nos municípios $A$ e B a diferença entre as categorias foi maior, quando comparada àquela do município $\mathrm{C}$ (Tabela 1 ). 
Tabela 1: Distribuição das categorias, segundo o município de procedência.

\begin{tabular}{l|c|c|c}
\hline \multicolumn{1}{c|}{ CATEGORIAS } & Município A & Município B & Município C \\
\hline & 323 & 285 & 229 \\
Competências técnico-científicas & $(77 \%)$ & $(77,5 \%)$ & $(58,1 \%)$ \\
& 97 & 83 & 165 \\
Relações interpessoais & $(23 \%)$ & $(22,5 \%)$ & $(41,9 \%)$ \\
Total & 420 & 368 & 394 \\
Relação entre categorias & $(100 \%)$ & $(100 \%)$ & $(100 \%)$ \\
\hline
\end{tabular}

A distribuição das duas categorias em função do sexo, em cada município, indica que Competências Técnico-científicas foram mais citadas pelos adolescentes de ambos os sexos. No entanto, em dois dos três municípios (Municípios A e C), essa categoria foi mais frequente entre os sujeitos do sexo feminino (Tabela 2).
Pelo teste do qui-quadrado, encontrouse diferenças significativas para o município $A(p=0,00000)$ e para o $B(p=0,0003)$. Nesses dois municípios, foram verificadas elevadas diferenças entre as categorias, sendo que no município $A$ ela foi maior entre os sujeitos do sexo feminino e no município $B$ foi entre os do sexo masculino (Tabela 2).

Tabela 2: Frequência das categorias, segundo sexo e município de procedência.

\begin{tabular}{l|c|c|c|c|c|c}
\hline \multirow{2}{*}{ Categorias } & \multicolumn{2}{|c|}{ Município A } & \multicolumn{2}{c|}{ Município B } & \multicolumn{2}{c}{ Município C } \\
\cline { 2 - 7 } & Fem. & Mas. & Fem. & Mas. & Fem. & Mas. \\
\hline \multirow{2}{*}{ Competências Técnico-Científicas } & 199 & 124 & 146 & 139 & 119 & 110 \\
$(85 \%)$ & $(67 \%)$ & $(70,5 \%)$ & $(86 \%)$ & $(60,7 \%)$ & $(55,6 \%)$ \\
\hline Relações Interpessoais & 35 & 62 & 61 & 22 & 77 & 88 \\
& $(15 \%)$ & $(33 \%)$ & $(29,5 \%)$ & $(14 \%)$ & $(39,3 \%)$ & $(44,4 \%)$ \\
\hline TOTAL & 234 & 186 & 207 & 161 & 196 & 198 \\
\hline & $(100 \%)$ & $(100 \%)$ & $(100 \%)$ & $(100 \%)$ & $(100 \%)$ & $(100 \%)$ \\
Relação entre categorias & & & & & & $\mathbf{1 , 5 4}$ \\
\hline
\end{tabular}

Considerando-se a ordem de evocação das categorias, verificou-se um comportamento similar entre os adolescentes dos três municípios. Em primeira ordem, o percentual de evocações enunciadas foi de $53,9 \%(n=637)$ e em segunda 46,1\% ( $n=545)$.

A categoria Relações Interpessoais, em primeira ordem de evocação, obteve
$50,1 \%(n=173)$ das citações e, em segunda ordem, 49,9\% ( $n=172)$ indicando uma queda de $0,2 \%$. Quanto à categoria Competências Técnico-científicas, em primeira ordem foram $55,4 \% \quad(n=464)$ e em segunda ordem $44,6 \% \quad(n=373)$, havendo uma queda de $10,8 \%$. Não houve associação significativa $(p=0,0971)$. 


\section{DIscussão}

A imagem que se tem do cirurgiãodentista pode influenciar no cuidado odontológico. Uma imagem negativa pode distanciar o profissional do paciente e levar à aquisição de atitudes negativas para com os procedimentos de atenção à saúde. ${ }^{14} \mathrm{Em}$ especial, quando o paciente se encontra na fase da adolescência, pois nesse período os jovens começam a ter controle sobre suas ações e desenvolver atitudes que podem afetar seus estilos de vida. ${ }^{15}$

É frequente observar, entre os adolescentes, atitudes de rebeldia, resistência a conselhos e percepção de não susceptibilidade ou vulnerabilidade a doenças. . $^{1,-9,16}$ Portanto, faz-se necessário considerar a atenção à saúde de adolescentes como primordial. O cirurgiãodentista, com os demais profissionais da área da saúde, pode colaborar no sentido de fazer que $o$ adolescente reflita sobre suas condutas e acerca das possibilidades de escolhas mais saudáveis.

Entre os adolescentes pesquisados encontrou-se um comportamento positivo, no que se refere à efetivação da consulta odontológica, uma vez que a maioria, nos três municípios, afirmou ter realizado sua última consulta a menos de um ano, tendo como segundo motivo mais citado a rotina/prevenção. Condição essa que vem ao encontro de outras pesquisas com adolescentes de diferentes contextos. ${ }^{17-19}$

$O$ fato de esses adolescentes terem acesso a serviços odontológicos e praticarem consultas por rotina, indiscutivelmente permite-nos dizer que estas são condutas que contribuem para amenizar condições desfavoráveis de saúde, pois a consulta odontológica com periodicidade e frequência adequadas possibilita o tratamento precoce e a prevenção de doenças. ${ }^{17,19}$ Esse quadro nos leva a crer que esses sujeitos estão bem familiarizados com o profissional da odontologia e seus procedimentos o que, certamente, Ihes permitiu identificar características que acreditam ser fundamentais ao cirurgiãodentista.

Nesse sentido, observou-se que os participantes enfatizaram as características vinculadas à categoria Competências Técnicocientíficas. Provavelmente, as evocações sobre Relações Interpessoais não tenham sido as mais citadas em decorrência do perfil dos pesquisados. A maioria, nos três municípios, havia efetuado sua última consulta odontológica a menos de um ano e uma parcela expressiva costuma efetivá-la em clínicas particulares. Além do mais, tem-se o aspecto de que os três municípios possuem um alto IDH, que pode estar contribuindo para com o acesso aos serviços de saúde, tanto privados quanto públicos, com regularidade. Acredita-se, então, que, para esses sujeitos, os vínculos entre paciente e profissional já estejam sedimentados, que o relacionamento humano já faça parte da rotina de atendimento. Talvez, por isso, os aspectos empáticos tenham sido menos apontados do que o observado em outros estudos, 1,13,20,21 deslocando-se o foco para os aspectos relacionados à tecnologia leve-dura, que se refere ao conhecimento técnico-científico, e à tecnologia dura, que se reporta ao uso de equipamentos tecnológicos, às normas e às estruturas organizacionais. ${ }^{22}$

Outro fator que pode explicar a valorização das competências técnicocientíficas refere-se ao motivo mais citado para a consulta, qual seja o tratamento ortodôntico. Esse tratamento, por envolver diferentes tipos de aparelhos e técnicas, pode estar exercendo influência na percepção dos adolescentes. É preciso lembrar que adolescentes em tratamento ortodôntico costumam expressar atitudes positivas em relação a esse tratamento e trocar informações sobre os procedimentos a que estão sendo submetidos, bem como estão 
sujeitos à influência das mídias sociais que, em geral, supervalorizam o aparato odontológico. ${ }^{4}$

Diferentes autores ${ }^{13,15,20-25}$ destacam que as expectativas e percepções dos pacientes para com a atenção odontológica estão menos relacionadas à competência técnica e têm mais a ver com as atitudes e habilidades de comunicação entre o profissional e o paciente. Muito embora tenhamos encontrado entre os participantes desta pesquisa uma situação inversa, ficou evidente que esses adolescentes não desconsideram as características vinculadas à interação profissional-paciente. Elas são mencionadas em menor frequência do que os aspectos técnico-científicos, porém, tendem a se manter quando de primeira para segunda ordem de evocação.

Desse modo, pode-se afirmar que esses adolescentes almejam ser atendidos por um cirurgião-dentista competente tecnicamente, mas que lhes escute, que tenha uma atitude solidária e amigável, que explique opções de tratamento e procedimentos e que inspire confiança. A capacidade para se comunicar e as competências interpessoais são elementos essenciais à prática odontológica. ${ }^{1,13,20,23}$ Os trechos a seguir, que foram retirados do material produzido pelos adolescentes, demonstram claramente as expectativas dos pesquisados:

...ele deve terminar a consulta com excelência e ser sempre simpático... (S121, Mun. A)

O dentista tem que saber o que está fazendo. Tem que fazer um serviço bem feito. Gente boa, simpático... (S84, Mun. A)

Não adianta ser só inteligente, tem que ser simpático e que passe confiança... (S147, Mun. B)

Gentil com o paciente e ter conhecimento especializado... (S38, Mun. B)

... qualificação, muito bom no que faz e gostar do que faz... (S5, Mun. C)

Ter certificado completo, ser gentil, ser profissional... (S32, Mun. C)
O estabelecimento de vínculos entre cirurgião-dentista e paciente e o conhecimento técnico-científico do profissional, inquestionavelmente, devem ser valorizados. ${ }^{15,20-25}$ Contudo, outras características, que são os chamados atributos não clínicos do trabalho odontológico, também, são necessárias a um exercício profissional de qualidade. Aspectos como qualidade de instalações e equipamentos, ambiente agradável, preços e prazos oferecidos, dias e horários de atendimentos são indicadores tangíveis para o usuário de serviços odontológicos que precisam ser considerados pelos profissionais.

Uma estrutura física agradável e limpa, profissional e equipe com indumentária asseada conferem um modo mais acolhedor e humano, favorecendo, assim, a qualidade dos serviços odontológicos. Estes são itens que costumam ser observados pela clientela. ${ }^{13,25-27}$ Os adolescentes pesquisados, também, destacaram esses aspectos, como se pode ler nos trechos a seguir que foram transcritos dos instrumentos de coleta de dados.

Tem que estar com os aparelhos sempre esterilizados e o consultório limpo... (S51, Mun. A)

Tem que ter um ambiente agradável... (S96, Mun. A)

Precisa ser pontual. Saber usar seus instrumentos de trabalho... (S17, Mun. B)

Ele tem que ter um consultório com a higiene necessária, tem que ter pontualidade na hora e organizado... (S118, Mun. B)

Tem que ter um lugar limpo e com bons equipamentos... (S73, Mun. B)

Precisa também ter uma boa aparência... (S98, Mun. C)

Percebe-se que os adolescentes integrantes da pesquisa, embora procedentes 
de três contextos socioculturais distintos, manifestaram um comportamento similar no sentido de que indicam com maior frequência e em primeiro plano as características relativas aos aspectos de competência técnico-científica do profissional e condições de trabalho. Muito embora em um dos três grupos tenha sido encontrada uma diferença menor dessa categoria em relação à categoria Relações Interpessoal, acredita-se que a similaridade do comportamento possa ser explicada pela influência da mídia e dos pares, uma vez que a busca pela identificação com modelos e o compartilhamento de ideias com o grupo são características muito próprias dessa fase do desenvolvimento humano, as quais exercem influências sobre o comportamento das pessoas. ${ }^{4,10,14}$ Com relação às diferenças detectadas em função do sexo dos participantes, a literatura aponta que, além da condição socioeconômica e das crenças, o gênero é um fator que pode definir diferenças no modo de agir e pensar. ${ }^{7,10}$

Esses adolescentes, independentemente do município de origem e do sexo, esperam ser atendidos com base no princípio da integralidade, mediante um processo que requer o envolvimento dos aspectos pertinentes às três tecnologias do trabalho em saúde que são apontadas por Merhry e Franco, ${ }^{22}$ quais sejam: tecnologia leve-dura (conhecimento científico), tecnologia dura (aparato estrutural) e tecnologia leve (sensibilidade dos profissionais). Portanto, o profissional deve estar capacitado não somente do ponto de vista científico e técnico, mas também precisa apresentar um perfil crítico, humanístico e ético. ${ }^{15,20,21,23,26}$

A adolescência é um período crítico para a internalização de valores, atitudes e comportamentos, os quais tendem a permanecer ao longo da vida adulta. Assim, a importância de se estimular os jovens a adotarem hábitos de vida saudável é inegável e nesse sentido é imprescindível a participação do cirurgião-dentista. O cirurgião-dentista deve conhecer as expectativas de sua clientela e procurar estabelecer vínculos que favoreçam o processo de cuidados para com a saúde. Estabelecer a qualidade do atendimento, com base nas expectativas desses jovens, pode ampliar o acesso dos adolescentes aos serviços prestados seja em consultórios particulares ou nas Unidades Básicas de Saúde. ${ }^{5}$

Finalizando, no que concerne aos limites do estudo, temos a considerar que não é possível extrapolar seus resultados, pois a percepção sobre determinado fenômeno é influenciada por fatores sociais inerentes a cada contexto e é dinâmica, devendo ser constantemente revisada dentro de um mesmo grupo. O modo como os adolescentes pesquisados expressaram sua visão a respeito das características essenciais ao cirurgião-dentista representa seus valores e expectativas, que pode, ou não, ser similar ao modo de interpretação de outros grupos de adolescentes.

Não obstante tal consideração, destacase que seus resultados oferecem indicadores que podem servir de subsídios a estudos com outros grupos de adolescentes. Acreditase, também, que as reflexões decorrentes deste trabalho podem fomentar as constantes discussões sobre a formação do cirurgiãodentista, que se inicia na graduação e deve se estender por toda a vida profissional.

\section{CONSIDERAÇÕES FINAIS}

Com base na análise dos dados obtidos neste estudo, conclui-se que a visão desse grupo de adolescentes, quanto às características essenciais do cirurgião-dentista para o exercício profissional, está fortemente vinculada à excelência do fazer técnicocientífico, para os sujeitos de ambos os 
sexos e nos três municípios de origem, sem, no entanto, excluírem as características que envolvem as relações interpessoais.

\section{Agradecimentos}

Ao Programa de Iniciação Científica Artigo 170/Governo do Estado de Santa Catarina.

\section{REFERÊNCIAS}

1. Bottan ER, Vitoretti AJ, Santi DG, Silveira EG. Percepção de adolescentes sobre as competências essenciais ao cirurgião-dentista. Arq. Odontol. 2015; 51 (3): 145-51.

http://revodonto.bvsalud.org/pdf/aodo/v51n3/ a04v51n3.pdf

2. Silva RT, Freixinho ABS, Miasato JM. Verificação do conhecimento e hábitos de saúde bucal em adolescentes de uma escola particular. Rev. odontol. Univ. Cid. São Paulo 2012; 24 (1): 19-25. http://publicacoes.unicid.edu.br/index. php/revistadaodontologia/article/view/351

3. Souza NT, Moreira RFAS, Miranda MS. Evasão do atendimento odontológico pelos adolescentes. Adolesc Saúde 2013; 10 (2): 63-6.

http://www.adolescenciaesaude.com/detalhe artigo. asp? id $=371$

4. Vazquez FL, Cortellazzi KL, Gonçalo CS, Bulgarelli JV, Guerra LM, Tagliaferro ESP et al. Estudo qualitativo sobre as justificativas de adolescentes para a não adesão ao tratamento odontológico. Ciênc. saúde colet. 2015; 20 (7): 2.147-56. Doi: 10.1590/1413-81232015207.04502014.

5. Brasil. Ministério do Planejamento, Orçamento e Gestão. Ministério da Saúde. Instituto Brasileiro de Geografia e Estatística. Pesquisa Nacional de Saúde do Escolar - 2012. Rio de Janeiro: IBGE; 2013. https://biblioteca.ibge.gov. br/visualizacao/livros/liv64436.pdf

6. Boeira GF, Salas MMS, Araújo DC, Masotti AS, Correa MB, Demarco FF. Factors influencing dental appearance satisfaction in adolescents: a cross-sectional study conducted in Southern of Brazil. Braz. J Oral Sci. 2016; 15
(1): 8-15. Doi: http://dx.doi.org/10.20396/bjos. v15i1.8647091.

7. Costa SMB, Machado MTC. O corpo e a imagem corporal em adolescentes: perspectivas a partir do cuidado integral à saúde. Adolesc Saude 2014; 11 (2): 19-24.

8. Sawyer SM, Afifi RA, Bearinger LH, Blakemore SJ, Dick B., Ezeh AC, Patton GC. Adolescence: a foundation for future health. Lancet 2012; 379 (9826): 1.630-40. Doi: 10.1016/S01406736(12)60072-5.

9. Viner RM, Ozer EM, Denny S., Marmot M., Resnick M., Fatusi A. et al. Adolescence and the social determinants of health. Lancet 2012; 379 (9826): 1.641-52. Doi: http://dx.doi.org/10.1016/ S0140-6736(12)60149-4.

10. Zamboni GLP, Lima RL, Duarte DA, Sant'Anna GR. Percepções, conhecimentos e representações de saúde bucal em adolescentes de escolas públicas e privadas do município de Atibaia, SP. RFO UPF 2015; 20 (2): 179-86. Doi: 10.5335/rfo.v20i2.4693.

11. Brasil. Ministério da Saúde. Saúde do adolescente e do jovem. Caderneta de saúde do adolescente. Brasília: Ministério da Saúde; 2014. http://bvsms.saude.gov.br/bvs/publicacoes/caderneta_saude_adolescente_feminina.pdf

12. Nóbrega SM, Coutinho MPL. O teste de associação livre de palavras. In: Coutinho MPL, Oliveira FB, Fortunato ML. Representações sociais: abordagem interdisciplinar. João Pessoa: Editora Universitária/UFPB; 2003, p. 67-77.

13. Bottan ER, Dall Oglio J.; Silveira EG, Araújo, SM. Cirurgião-dentista ideal: perfil definido por crianças e adolescentes. RSBO 2009; 6 (4) 381-6. https://www.redalyc.org/ pdf/1530/153012921007.pdf

14. Barbosa RC, Carvalho RB, Santana MIP, Surdini JP, Barbosa GPS, Almeida ER. Percepção de escolares sobre o cirurgião-dentista: análise antes e depois de práticas educativas. Rev. Bras. Pesq. Saúde 2015; 17 (1): 81-6. Doi: http:// dx.doi.org/10.21722/rbps.v17i1.12453.

15. Al-Hussyeen AJ. Factors affecting utilization of dental health services and satisfaction among adolescent females in Riyadh City. Saudi Dent J 2010; 22 (1): 19-25. DOI: 10.1016/j. sdentj.2009.12.004 
16. Raphael D. Adolescence as a gateway to adult health outcomes. Maturitas 2013; 75 (2): 13741. Doi: 10.1016/j.maturitas.2013.03.013.

17. Gibilini C., Esmeriz CEC, Volpato LF, Meneghim ZMAP, Silva DD, Sousa MLR. Acesso a serviços odontológicos e auto-percepção da saúde bucal em adolescentes, adultos e idosos. Arq. Odontol. 2010; 46 (4): 143-223. https://periodicos.ufmg.br/index.php/arquivosemodontologia/ article/view/3548

18. Kavand G., Broffitt B., Levy SM, Warren JJ. Comparison of dental esthetic perceptions of young adolescents and their parents. J Public Health Dent. 2012; 72 (2): 164-71. Doi: 10.1111/j.1752-7325.2011.00306.x.

19. Oliveira RCN, Souza JGS, Oliveira CC, Oliveira LFB, Pelino JEP, Martins AMEBL, Almeida ER. Acesso a informações sobre como evitar problemas bucais entre escolares da rede pública de ensino. Ciênc. saúde coletiva 2015; 20 (1): 85-94. Doi: http://dx.doi.org/10.1590/141381232014201.00032014.

20. Bottan ER, Silveira EG, Odebrecht CMR, Araújo SM, Farias MMAG. Relação entre ansiedade ao tratamento dentário e caracterização do dentista ideal: estudo com crianças e adolescentes. Rev. port. estomatol. cir. maxilofac. 2010; 51 (1): 19-23. Doi: http://dx.doi.org/10.1016/ S1646-2890(10)70081-9.

21. Sbaraini A., Carter SM, Evans RW, Blinkhorn A. Experiences of dental care: what do patients value? BMC Health Serv Res 2012; 12 (177). Disponível em: <http://www. biomedcentral. com/1472-6963/12/177>. Doi: 10.1186/14726963-12-177.

22. Merhy EE, Franco TB. Por uma composição técnica do trabalho centrada nas tecnologias leves e no campo relacional. Saúde em Debate 2003; 27 (65): 316-23.

https://www.pucsp.br/prosaude/downloads/territorio/composicao_tecnica_do_trabalho_emerson_merhy_tulio_franco.pdf

23. Broder HL, Janal M., Mitnick DM, Rodriguez JY, Sischo L. Communication skills in dental students: new data regarding retention and generalization of training effects. J Dent Educ. 2015; 79 (8): 940-8. https://pubmed.ncbi.nlm. nih.gov/26246533/

24. Churchill JL. What's a dentist to do? Empathy, part two. Northwest Dent. 2010; 89 (5) 43-4. https://www.researchgate.net/publication/285590924_20_2012_142_20-

25. Figueiredo MC, Faustino-Silva DD, Faneze JL, Gass EL, Correia KV, Silva LA. El papel del odontólogo como facilitador de la relación com el paciente adolescente: una revisión de literatura. Odontoestomatología 2010; XII (14): 15-28. http://www.scielo.edu.uy/scielo.php?script=sci_ arttext\&pid=S1688-93392010000100003

26. Ramseier CA, Wolf CA. The image of the dentist. Part 2: The ideal dentist. Schweiz Monatsschr Zahnmed 2012; 122 (3): 219-29. https://www. teses.usp.br/teses/disponiveis/23/23148/tde13042013-125024/publico/TeresaMariadaPonteVersaoCorrigida.pdf

27. Bordin D., Fadel CB, Moimaz SAS, Garbin CAS, Saliba NA. Considerações de profissionais e usuários sobre o serviço público odontológico: um aporte para o planejamento em saúde. Rev. APS 2016; 19 (2): 221-9. https://periodicos.ufff. br/index.php/aps/article/view/15732

Submetido em: 30-1-2017

Aceito em: 15-10-2020 